\title{
Employing Numerical Methods to Computational Biological System Problems
}

\author{
Sukumar Senthilkumar* \\ School of Computing Science and Engineering, Vellore Institute of Technology-University, India
}

Indeed numerical methods in biological modeling play a vital role towards solving many of the biological problems which gained considerable popularity and importance, mainly owing to its demonstrated applications in numerous diverse and widespread fields in science and engineering. It is significant to point out that computational and systems biology (CSB) encompasses an interdisciplinary approach that harnesses the power of numerical computation and systems-level analyses to formulate and solve critical biological problems which involves ordinary differential equations. Moreover, in CSB the modeling and mathematical investigations of biological systems using sophisticated computational techniques is an exciting, innovative field of multidisciplinary research that addresses the complex challenges in understanding the behavior of biological systems and human diseases. Researchers concentrate much on modeling dynamical systems in time and space, for example chemical kinetics, interacting and competing species, pattern formation in development. Numerical methods and algorithms are employed to solve particular biological problems arising in population dynamics, chemical reaction kinetics and pattern formation (reaction diffusion systems) etc. Classification of mathematical problems and choosing appropriate numerical methods are important to solve biological problems (predator-prey model) efficiently through sophisticated application software. Moreover, cognitive skills are necessary to understand the concepts and theory related to numerical methods and use of numerical approaches to interpret data from a wide range of different areas and to aid their critical interpretation of such data are significant.

It is known from literature that many standard biochemistry texts provide thorough derivations of Ordinary Differential Equations (ODEs) for both simple and complex reactions. In fact, ODE based modeling is the most common simulation approaches in computational systems biology, reflecting both its rigor and adaptability. Analytic solutions of most ordinary differential equations cannot be obtained easily for some cumbersome problems. As a consequence, approximate and numerical techniques are playing an important role in identifying the solution behavior of such fractional equations and exploring their applications. i.e., Simple ODEs may have exact solutions. But on the other hand, most complex ODEs do not have exact solutions and must be solved numerically. Numerical integrators for Newton and Gauss methods, utilize linear approximations of smooth curves over small time intervals to compute subsequent values of reactant concentrations. Improving the accuracy of these linear estimates may need using smaller time intervals, leading to computationally intense processes that use considerable machine time. There is a vast literature on different techniques and algorithms which are developed to improve the speed and computational accuracy of these approximate techniques on numerical integration, and the standard methods for integrating ordinary differential equations, namely Euler's method, explicit RungeKutta algorithms and implicit Rune-Kutta methods (for so-called "stiff" differential equations) and their adaptive versions (Runge-KuttaFehlberg), multi-step methods (Adams-Bashforth-Moulton) and so on are described in many introductory texts on differential equations. However, they have been implemented in numerous software packages that are both highly sophisticated and readily available for easier access.

For instance, delay differential equations can reduce the computational effort similarly; systems of ODEs have been used to mathematically model a wide variety of processes including metabolic pathways and genetic regulatory circuits. Computational system biology includes parameter estimation (inverse problems, model calibration) in biochemical pathways, optimal experimental design (optimal dynamic experiments) for systems biology, dynamic optimization (optimal control) of biosystems and bioprocesses, nonlinear analysis of biochemical reaction networks and robust control of diffusion-reaction systems, with applications in biosystems. Finally, computational systems biology simulation software packages (ODE, PDE, SSF, Petri Net, DCA and ABM) are available to solve biological problems which arise in many fields of biological domain.

*Corresponding author: Sukumar Senthil Kumar, School of Computing Science and Engineering, Vellore Institute of Technology-University, Vellore-632014, Tamilnadu, India, Tel: 9489107683; E-mail: senthilkumar@vit.ac.in, ssenthilkumar1974@yahoo.co.in

Received January 22, 2014; Accepted January 24, 2014; Published January 27, 2014

Citation: Senthilkumar S (2014) Employing Numerical Methods to Computational Biological System Problems. J Comput Sci Syst Biol 7: e107. doi:10.4172/ jcsb.1000e107

Copyright: $\odot 2014$ Senthilkumar S. This is an open-access article distributed under the terms of the Creative Commons Attribution License,which permits unrestricted use, distribution, and reproduction in any medium, provided the original author and source are credited. 